\title{
Reflexões acerca da assistência e ensino à saúde de populações tradicionais: inclusão e cenários
}

\author{
Reflections on the assistance and health education of traditional populations: inclusion and \\ scenarios
}
Reflexiones acerca de la asistencia y enseñanza a la salud de poblaciones tradicionales: inclusión y escenarios

Nádile Juliane Costa de Castro $^{1 *}$, Jéssica Bruna Gomes Teixeira², Juliana Pereira Pinto Cordeiro $^{3}$, Dayanne de Nazaré dos Santos ${ }^{4}$.

\begin{abstract}
RESUMO
Objetivo: Este artigo identifica a inclusão de atividades de ensino para assistência à saúde de populações tradicionais, nos cursos de graduação em medicina de universidades e faculdades da região Norte do Brasil a fim de mediar reflexões acerca do tema. Métodos: Pesquisa realizada por método misto, longitudinal, com investigação de coleta de dados com abordagem de estudo de casos múltiplos, por meio de base de dados do Ministério de Educação e Cultura, ano base 2018. Para o constructo foram definidos descritores a serem pesquisados nas matrizes e ementas dos projetos políticos pedagógicos. O parâmetro base para inclusão dos dados se delimitou nos termos: populações tradicionais; populações amazônicas; saúde indígena; saúde de grupos étnicos e populações remanescentes de quilombo nos textos do projeto pedagógico (matrizes curriculares e ementas). Resultados: Foram identificados 20 cursos, sendo nove públicos e onze privados, deste apenas três apresentaram disciplinas dentro da característica investigada, sendo estas ministradas no estado do Amazonas e Acre. Conclusão: É notória a ausência de disciplinas curriculares sobre populações tradicionais nos currículos de Medicina. Os resultados possibilitam entender também que há um esvaziamento dos conteúdos sociais e humanísticos nas disciplinas de base na maioria dos currículos.
\end{abstract}

Palavras-chave: Ensino, Populações tradicionais, Projeto pedagógico, Amazônia, Saúde coletiva.

\begin{abstract}
Objective: This article identifies the inclusion of teaching activities for the health care of traditional populations, in undergraduate courses in medicine of universities and colleges of the Northern region of Brazil in order to mediate reflections on the subject. Methods: A longitudinal, mixed research with data collection investigation with a multiple case study approach, using a database of the Ministry of Education and Culture, base year 2018. For the construct, descriptors were defined to be searched in the matrices and menus of pedagogical political projects. The basic parameter for inclusion of the data was delimited in terms: traditional populations; Amazon populations; indigenous health; health of ethnic groups and remaining populations of quilombo in the texts of the pedagogical project (curricular matrices and menus). Results: Twenty courses were identified, of which nine were public and eleven were private, of which only three presented disciplines within the investigated trait, being these taught in the state of Amazonas and Acre. Conclusion: The absence of curricular subjects on traditional populations in medical curricula is notorious. The results also allow us to understand that there is a depletion of social and humanistic contents in the basic subjects in most curricula.
\end{abstract}

Key words: Teaching, Traditional populations, Pedagogical project, Amazon, Collective health.

\footnotetext{
1 Universidade Federal do Pará, Belém-PA. *E-mail: nadiledecastro@hotmail.com

${ }^{2}$ Faculdade Estácio, Castanhal, PA.

${ }^{3}$ Faculdade da Amazônia, Belém, PA.

${ }^{4}$ Faculdade Brasil Amazônia, Belém, PA.
}

SUBMETIDO EM: 2/2019 


\section{RESUMEN}

Objetivo: Este artículo identifica la inclusión de actividades de enseñanza para asistencia a la salud de poblaciones tradicionales, en los cursos de graduación en medicina de universidades y facultades de la región Norte de Brasil a fin de mediar reflexiones sobre el tema. Métodos: Investigación realizada por método mixto, longitudinal, con investigación de recolección de datos con abordaje de estudio de casos múltiples, por medio de base de datos del Ministerio de Educación y Cultura, año base 2018. Para el constructo se definieron descriptores a ser investigados en las matrices y menús de los proyectos políticos pedagógicos. El parámetro base para la inclusión de los datos se delimitó en los términos: poblaciones tradicionales; poblaciones amazónicas; salud indígena; salud de grupos étnicos y poblaciones remanentes de quilombo en los textos del proyecto pedagógico (matrices curriculares y menús). Resultados: Se identificaron 20 cursos, siendo nueve públicos y once privados, de éste sólo tres presentaron disciplinas dentro de la característica investigada, siendo estas ministradas en el estado de Amazonas y Acre. Conclusión: Es notoria la ausencia de disciplinas curriculares sobre poblaciones tradicionales en los currículos de Medicina. Los resultados posibilitan entender también que hay un vaciamiento de los contenidos sociales y humanísticos en las disciplinas básicas en la mayoría de los currículos.

Palabras clave: Enseñanza, Poblaciones tradicionales, Proyecto pedagógico, Amazonia, Salud colectiva.

\section{INTRODUÇÃO}

A Amazônia apresenta diversidade étnica e cultural evidenciada por diversos autores (COCKS M, 2006; SIMONIAN LTL, 2007; BALÉE W, 1993). Este contexto deve ser considerado quando de aspectos que instituem ações diretas e indiretas nestas populações, incluso nesse cenário os serviços de saúde. Ao considerar estes fatos e compreendendo a organização do Sistema de Saúde Único (SUS) com destaque para as demandas da Atenção Básica, identifica-se as populações tradicionais inclusas nestas dinâmicas.

Como aporte para essa realidade foram instituídos dispositivos legais que garantem que os serviços de saúde as populações específicas. Cita-se a Lei 9.836/99 que cria o Subsistema indígena, e as portarias 290/2013, 992/2009, 2866/2011, que são de ordem respectivamente da garantia de serviços de Unidades Fluviais, Política de atenção à população negra, e Política nacional de saúde integral das populações do campo e da floresta. Todas estas estão em conformidade as propostas do SUS e colaboram com a realidade da atenção primária à saúde das populações da Amazônia (BRASIL, 1999; BRASIL, 2011; BRASIL, 2013; BRASIL, 2015).

Com o objetivo de alcançar estas populações e as diretrizes do SUS, a infraestrutura da atenção primária em saúde é consolidada por equipes multidisciplinares, onde se inclui o médico. Considerando estes fatos, é perceptível a importância deste profissional na equipe e nas rotinas destes serviços, o que demonstra claramente a importância do mesmo está inserido no contexto social desse processo. Contudo, o que se observa são deficiências nessa área de atuação por consequência da formação acadêmica (BARBOZA JS, et al 2016).

Nos últimos anos mudanças nos currículos de formação médica vem sendo identificadas. As Diretrizes Curriculares Nacionais do Curso de Graduação em Medicina (DCN) vêm corroborar fatos já elencados como a visão crítica, reflexiva e humanística, assim como da determinação social e integralidade da saúde do indivíduo. Ainda, a própria legislação destaca a diversidade étnico-racial a serem consideradas na atenção à saúde (BRASIL, 2014).

Por meio destes fatos, pelo contexto das competências médicas, previstas pelas DCN e seus delineamentos, o aprendizado deve articular cognição com atividades psicomotoras e socioafetivas. Tal ato se efetivaria pela execução de ações durante o aprendizado que incluíssem as diversas dimensões socioculturais, político-econômicas e histórico-geográficas. Nestes modos, se alcançaria então a formação profissional humanística em toda sua amplitude, instituindo o previsto nas DCN (SANTOS IWS, 2011). 
Partindo deste cenário, é necessário entender que as atividades executadas na Rede Básica de Saúde se constroem pela composição final de territórios, sociedade e rotinas das populações atendidas pelos serviços. Entretanto, não é essa a realidade percebida e/ou idealizada pelos acadêmicos, o que favorece que o exercício da profissão não seja o esperado. Justamente por essas asserções que é necessário realizar atividades problematizadoras que incluam todo a composição do cenário (AZEVEDO BMS, 2013).

O trabalho se justifica pela percepção da importância da inserção deste tema nos projetos pedagógicos, a fim de construir habilidades inerentes a esse campo de conhecimento. Este artigo identifica a inclusão de atividades de ensino para assistência à saúde de populações tradicionais, nos cursos de graduação em medicina de universidades e faculdades da região Norte do Brasil a fim de mediar reflexões acerca do tema.

\section{MÉTODOS}

Estudo descritivo e exploratório, de natureza mista, pois fora realizada a associação de pesquisa qualitativa e quantitativa ao longo da dinâmica metodológica (CRESWELL JW, 2012). Tais delineamentos possibilitaram o compartilhamento das informações de modo que estas se complementassem ao longo do processo de pesquisa, realizando a construção do estudo (YIN RK, 2015). Tais evidências se corroboram pelo fato de que o estudo também permitiu a transformação dos dados ao longo de seu processo, o que justamente a caracterizou como uma pesquisa aninhada concomitante (CRESWELL JW, 2012).

Para amplitude das fontes fora escolhido o estudo de caso múltiplo, pois esta abordagem nos permitiu compreender como o objeto estudado se comportou nos diversos cursos registrados na plataforma eletrônica, compreendendo, portanto, que cada região representa uma unidade/caso (YIN RK, 2015). Assim sendo, foi definido como critérios de inclusão as instituições de ensino superior (IES) cadastradas nos estados da região norte do Brasil (Amapá, Acre, Amazonas, Pará, Rondônia, Roraima e Tocantins) no site oficial de dados do Cadastro Nacional de Cursos e Instituições de Ensino Superior Cadastro e-MEC (E-MEC) no ano base 2018 e das matrizes e projetos políticos pedagógicos das IES em cadastro. Por fim, o processo de investigação ocorreu entre os meses de janeiro a abril de 2018, de modo longitudinal a fim de identificar as características de cada unidade e conforme legislação vigente sobre divulgação de informações sobre IES.

Para o constructo da pesquisa foram realizados roteiros que incluíam as características essenciais para debate do objeto em estudo, com descritores a serem pesquisados nas matrizes e ementas dos projetos políticos pedagógicos. Nestes termos foram definidos: Tipo de serviço oferecido (público e particular), modelo de atuação (a distância e presencial), ausência ou presença de disciplina específica ou afim, matriz e projeto pedagógico disponível. O parâmetro base para inclusão dos dados se delimitou nos termos: populações tradicionais; populações amazônicas; saúde indígena; saúde de grupos étnicos e populações remanescentes de quilombo nos textos do projeto pedagógico (matrizes curriculares e ementas).

A coleta de dados fora realizada separadamente de acordo com os instrumentos definidos: roteiros para o E-MEC e busca de descritores nas ementas. Assim sendo, os dados qualitativos foram coletados por meio da busca de descritores nos projetos pedagógicos e categorizados a fim de serem transformados em dados quantitativos. Os dados quantitativos foram obtidos pela coleta direta de informações cedidas no site do EMEC. Estes foram somados com os dados qualitativos transformados em dados numéricos, sendo estes descritos e tabulados. Analisou-se as matrizes e projetos pedagógicos da IES participantes, onde foram realizados exploração do material, tabulação de dados dos casos isoladamente e posterior análise conjunta, com devida interpretação, no modelo de análise estatística.

Vale ressaltar que por se tratar de base de dados públicos e não envolver pesquisas com seres humanos, assim como de dados secundários destes, ratifica-se que este estudo não necessitou de apreciação de um comitê de ética em pesquisa. 


\section{RESULTADOS}

Na plataforma do E-MEC foram identificados o cadastro de 20 cursos vinculados a instituições públicas e/ou privadas, na modalidade presencial e a distância, conforme desenho do estudo. A Tabela 1 mostra as unidades pesquisadas considerando suas características individuais e conjuntura no universo estudado. Notase que o estado de Tocantins apresenta 5 cursos (25\%), seguido do Estado do Pará e Rondônia com 5 (25\%) cada, e logo em seguida o estado do Amazonas com três cursos (15\%), Acre dois cursos (10\%) e por último os estados do Amapá e Roraima com um curso cada (5\%).

No quesito cursos com disciplinas com temáticas sobre populações tradicionais da Amazônia, foram identificadas o total de três, sendo duas destas no estado do Amazonas (2/20 - 10\%) e uma no estado do Acre $(1 / 20-5 \%)$. Esta quanto à distribuição de Instituições públicas e privadas com disciplinas voltadas às populações tradicionais em suas respectivas matrizes e/ou projetos políticos pedagógicos, identificou-se todas como de iniciativa pública o que representa (3/9-33\%) nesta modalidade.

Os estados que apresentam IES com disciplinas, Amazonas (2/3 - 66,7\%) e Acre $(1 / 3-33,3 \%)$ são contrastantes com os demais estados da região. Assim sendo, Amapá, Pará, Rondônia, Roraima e Tocantins pela ausência de disciplinas específicas nos cursos oferecidos apresentam dados bem diferentes $(0 / 20-0 \%)$ dos demais $(3 / 20-15 \%)$.

Tabela 1 - Características dos cursos de Medicina na região Norte em relação ao ensino da saúde de populações tradicionais, 2018.

\begin{tabular}{cccccccccc}
\hline ESTADOS & AP & AC & PA & AM & RR & RO & TO $\begin{array}{c}\text { TOTAL } \\
\text { (N) }\end{array}$ & (\%) \\
\hline $\begin{array}{c}\text { Total de cursos } \\
\text { Com disciplina sobre }\end{array}$ & 1 & 2 & 4 & 3 & 1 & 4 & 5 & 20 & $100 \%$ \\
$\begin{array}{c}\text { populações tradicionais } \\
\text { Sem disciplinas sobre }\end{array}$ & 1 & 1 & 0 & 1 & 0 & 0 & 0 & 3 & $6 \%$ \\
populações tradicionais & 0 & 1 & 4 & 3 & 1 & 4 & 5 & 17 & $34 \%$ \\
$\begin{array}{c}\text { Presencial } \\
\text { À distancia }\end{array}$ & 1 & 2 & 4 & 3 & 1 & 4 & 5 & 20 & $40 \%$ \\
$\quad 0$ & 0 & 0 & 0 & 0 & 0 & 0 & 0 & $0 \%$ \\
$\begin{array}{c}\text { Públicos } \\
\text { Privados }\end{array}$ & 1 & 1 & 2 & 2 & 1 & 1 & 1 & 9 & $18 \%$ \\
$\begin{array}{c}\text { Disciplinas de antropologia à } \\
\text { saúde }\end{array}$ & 0 & 1 & 2 & 1 & 0 & 3 & 4 & 11 & $22 \%$ \\
$\begin{array}{c}\text { Sem informação de currículo } \\
\text { na internet }\end{array}$ & 0 & 1 & 0 & 0 & 0 & 1 & 0 & 2 & $4 \%$ \\
\hline \hline
\end{tabular}

Fonte: Cadastro Nacional de Cursos e Instituições de Ensino Superior Cadastro e-MEC (E-MEC) 2018.

Em relação ao título da disciplina ofertada e sua carga horária esta é definida por Saúde Indígena (2/3 $66,7 \%$ ) com a primeira com 75 horas, dividido em 15 horas teóricas e 60 horas práticas, e a segunda com 45 horas, sem demais definições e Rural e Indígena (1/3 - 33,3\%). Todas estas três disciplinas são ofertadas como optativas. 
Já em consonância do tema de modo interdisciplinar no ciclo de formação básica não fora identificado nenhuma disciplina de antropologia à saúde, porém foram identificadas Saúde coletiva, Políticas públicas e Atenção integral à saúde, e na formação profissional em regime de internato a disciplina saúde coletiva. Assim como ofertas de modo optativo como o caso de sociologia aplicada à saúde.

Sobre a disponibilidade dos dados de projetos pedagógicos e afins em plataformas, sites e redes de acesso virtual, duas IES não apresentam estas informações conforme Tabela 1. Do total de 20 unidades investigadas, isto representou $20 \%$ do total, sendo estes mesmos dados identificados como $18 \%$ das IES de iniciativa privada. Ainda no sentido de caracterização, não foram identificados casos de IES com modalidades à distância em nenhuma unidade estudada.

\section{DISCUSSÃO}

\section{As diretrizes curriculares e ações no ensino como papel transformador}

Em consonância de implementar mudanças que alcancem todas as necessidades evidenciadas nos diversos serviços, regiões e grupos populacionais, foram criadas as DCN. Estas preveem por meio da formação do médico implantar competências e habilidades específicas que possibilitem uma visão humanística dentro desse cenário. Justamente no Artigo 5econtra-se duas dessas características: "Lidar criticamente com a dinâmica do mercado de trabalho e com as políticas de saúde" e "Ter visão do papel social do médico e disposição para atuar em atividades de política e de planejamento em saúde" (BRASIL, 2014).

Por esses delineamentos é necessário entender que quando da pertinência de atividades disciplinares com conteúdo das políticas de saúde, possibilita-se transformar o aluno em ser político. Isto colabora para que desafios como da construção de um novo perfil profissional seja realizado, rompendo fronteiras para que um haja de fato transformação da prática profissional (BRASIL, 2001). Logo, entende-se que tal ação promove ativamente que desde a academia o futuro profissional seja um formador de opinião, sendo esta uma ação que ajude na sua tomada de decisão quando necessária.

As previsões descritas nas DCN são pertinentes quando de modo mais categórico envereda-se para assistências de grupos específicos (BRASIL, 2014). Afirma-se isto justaposto que quando do conhecimento das políticas específicas durante os anos iniciais de formação, possibilita-se a continuidade desse processo ao longo da formação do ciclo profissional (BARROSO et al., 2012). Por certo, instiga-se o discente a refletir sobre esses aspectos.

Uma das formas de alcançar tal patamar é a interdisciplinaridade. A integralidade do conhecimento do ser humano deve ser uma concepção interdisciplinar, pois, envolve aspectos de saúde, doença e trabalho, possibilitando uma abordagem de rompimento entre pesquisa e ensino-aprendizagem (BRASIL, 2001). Certamente, os currículos pesquisados têm se apoiado nessa perspectiva, pois foi encontrado eixos sobre humanidades, disciplinas de políticas públicas de saúde e ainda, de modo optativa, disciplinas de áreas sociais e humanas. Estes fatos colaboram para a construção de currículos integrados e com destaques para aspectos relevantes (BRASIL, 2001).

Essa constituição possibilita uma rede de colaboração entre ensino e assistência, e ainda mais, consegue avançar nos aspetos relacionadas as necessidades regionais. Deste modo, como previsto pelas DCN, os problemas das realidades tornam-se visíveis ao longo do processo de produção da aprendizagem em saúde, promovendo articulações entre disciplinas, o que favorece a promoção à saúde (BRASIL, 2001). Assim sendo, "Por todas essas razões, é muito importante, para uma formação de qualidade na área da saúde, que os estudantes possam vivenciar e, efetivamente, inserir-se no trabalho que ocorre nesses múltiplos cenários de prática da atenção à saúde" (BRASIL, 2001).

REAS/EJCH | Vol. 11 (6) | e556 | DOI: https://doi.org/10.25248/reas.e556.2019 Página 5 de 8 
Já em referência ao papel social e considerando os fatos elencados, é favorável para essa característica a efetivação de atividades que envolvam o saber pelas políticas (BARROSO et al., 2012). Também se pondera as referências pelas representações de estudo de casos para grupos específicos, conforme o debate desta pesquisa, como por exemplo, pela oferta de disciplinas que aprofundem esses conhecimentos, ainda que de modo optativo. Tais situações ressignificam o aprendizado, pois vão além da prática biomédica, pois, consideram seu papel de promotor de necessidades regionais e culturais, conferindo-o como promotor de uma saúde mais social (LIMA et al., 2003).

Ainda nessa conjuntura, há de se dizer que ações como projetos de extensão também são favoráveis. Contextos neste sentido já apontam como vivências extracurriculares instituem uma maior aproximação com a realidade, pois além de inserir a universidade neste processo, esta ação promove que os acadêmicos vivam novas experiências, e as realidades das comunidades que serão egressos, tornando-os mais críticos destas, pelo reconhecimento das necessidades. E ainda, qualifica o mesmo para o mercado de trabalho e principalmente para a atenção básica, que é fundamental dentro da saúde indígena (SILVA RP et al., 2015).

\section{Cenário de ensino da assistência à saúde de populações tradicionais}

Nas últimas décadas há um grande crescimento de políticas de saúde para populações específicas, e nesse ensejo incluem-se as populações tradicionais. Esta configuração nada mais é que a resposta as necessidades das demandas do SUS e suas diretrizes de base. Entretanto, este cenário não tem se implementado na formação acadêmica dos profissionais de saúde que prestarão assistência à saúde a estas populações, perceptível pelos achados da pesquisa.

Foi possível identificar que dos 20 cursos oferecidos na região Norte, há uma baixa representatividade desta assistência específica de modo disciplinar. Tal evidência é destacada pela identificação de apenas três disciplinas identificadas no estudo. É perceptível também pelos achados das disciplinas de tronco comum, que não há interesse notório por estruturar a formação pelas ciências sociais e de humanidades como é previsto pelas diretrizes nacionais do ensino médico. Isto fica claro quando não encontrado neste recorte disciplinas como antropologia ou sociologia da saúde, por exemplo.

Há, entretanto, de se revelar que ainda que se tenha encontrado disciplinas específicas em algumas unidades do estudo, todas estão alocadas como disciplinas optativas, o que demanda por esta configuração a possibilidade do acesso aos seus conteúdos pela não escolha do tema pelo discente.

Outro fator de destaque é a definição de seus conteúdos, com ênfase para saúde indígena nos três casos. Isto reflete que não há um direcionamento para este grupo, o que pode ser resultado das políticas de saúde instituídas no âmbito do SUS. Essa realidade, porém, demonstra que outras populações amazônicas podem ser suprimidas dentro do ensino de saúde, como as populações ribeirinhas e quilombolas.

O estudo também revela que os casos como do estado do Amazonas e Acre, são resultados pontuais dos grupos de pesquisas na região cadastrados no Diretório de Pesquisa do Conselho Nacional de Pesquisa. Pontualmente isto reflete em ações acadêmicas já realizadas, como o I Seminário de Enfermagem em Saúde Indígena da Amazônia, no ano de 2017 na Universidade Federal da Amazônia. Pertinentemente é preciso compreender que há no estado do Amazonas e no Estado do Acre uma significativa população indígena de acordo com o último CENSO nacional (IBGE, 2012).

Ainda, de modo contraditório pela realidade das demandas de populações indígenas, há 13 mil indígenas declarados, o Estado de Tocantins não apresenta nenhuma disciplina com populações tradicionais (IBGE, 2010). Esta situação é preocupante, haja vista que considerando a demanda desta população na região e o fato deste Estado possuir o maior número de cursos registrados na base do E-MEC, o ensino da assistência direcionada as necessidades destas populações parece não ser contemplada de modo disciplinar e nem nas disciplinas do ciclo básico de formação.

REAS/EJCH | Vol. 11 (6) | e556 | DOI: https://doi.org/10.25248/reas.e556.2019 Página 6 de 8 
Pelos aportes apontados alguns elementos devem ser considerados, como por exemplo a interdisciplinaridade em que pode ser tratado o tema. É claro e evidente que tais conteúdos podem estar sendo parcialmente contemplados por mecanismos de estudos de casos clínicos e por discussões ainda que com carga horária reduzida e não obrigatória em outros campos disciplinares como já destacados em outros estudos similares (DE CASTRO NJC et al., 2017). Vale ressaltar, portanto, que tais situações não são capazes de suprir a pluralidade do tema em todos os ciclos de vida e de cuidado a saúde.

Ressalta-se isto, pois, quando de disciplina prevista no currículo, a carga horária é definida e exclusiva para o constructo da temática, como encontrado nos três casos identificados. Isto possibilita que todos os aspectos de assistência à saúde destas populações sejam contemplados ou ao menos reconhecidos pelo discente, promovendo assim habilidades inerentes as necessidades destes grupos (CASTRO NJC, 2015; DE CASTRO NJC et al., 2017, COSTA et al., 2018).

De modo institucional pelo modelo de iniciativa, sucede entender que tais preocupações ainda são pelas IES públicas, como observado nos resultados desta pesquisa semelhantemente como na formação em Enfermagem (DE CASTRO NJC et al., 2017). Notadamente isto é revelado pelas constantes mudanças de projetos pedagógicos destes cursos nos últimos anos, a fim de atender as diretrizes do ensino médico, e pela própria demanda que as universidades atendem.

Contudo, isto parece ser contraditório também quando se considera o aumento significativo de cursos de medicina privado na última década nesta região. Estas têm utilizado cada vez mais os espaços públicos de saúde para o ensino médico, dividindo as demandas destes serviços com as universidades públicas. Versa então refletir se estas estariam preocupadas com as demandas de populações tradicionais, ainda que seus currículos não sejam pontuais quanto a isto. Este fato é algo que deve ser pertinentemente pesquisado.

Ainda que ocorram atividades extracurriculares em áreas e campos de conhecimento que podem ampliar as habilidades com populações tradicionais, como no caso do Estado de Amapá que prevê atividade extracurricular em sociologia, é necessário perceber que tais delineamentos são arriscados. Estes riscos ocorrem justamente pela carga horária reduzida, e pela possibilidade de não contemplar populações específicas pela pluralidade que atividades como estas preveem.

\section{CONSIDERAÇÕES FINAIS}

O estudou revelou que é notória a ausência de disciplinas curriculares sobre populações tradicionais nos currículos de Medicina na região norte, apontando que não há uma preocupação com as demandas regionais. Os resultados possibilitam entender que há um esvaziamento dos conteúdos sociais e humanísticos nas disciplinas de base na maioria dos currículos. Em contrapartida a isto, é necessário entender que 0 reconhecimento das políticas individuais nos currículos dos cursos de medicina amplia as habilidades médicas e qualificam o profissional de saúde para as redes de saúde do SUS específicas, e demandas regionais. Esta iniciativa também possibilita que durante a formação médica, o discente reflita sobre o cuidado destas populações, tendo a possibilidade de ser um potencial colaborador para os princípios da equidade e solidariedade.

\section{REFERÊNCIAS}

1. AZEVEDO BMS, FERIGATO S, SOUZA TP, CARVALHO SR. A formação médica em debate: perspectivas a partir do encontro entre instituição de ensino e rede pública de saúde. Interface-Comunicação, Saúde, Educação, 2013;17(44):187-199.

2. BALÉE W. Indigenous transformations of Amazonian forests: na example from maranhão, Brazil. L'Homme, 1993; 33(126):231-254. 
3. BARBOZA JS, LEMOS ABS, BARREIRO BFG, PEIXOTO GBM. Relatos médicos sobre formação acadêmica e trabalho na Estratégia de Saúde da Família. Revista Brasileira de Medicina de Família e Comunidade, 2016;11(38):1-8.

4. BARROSO MGT, VIEIRA, NFC, VARELA, ZM. Ensino de educação em saúde, interdisciplinaridade e políticas públicas. Revista Brasileira em Promoção da Saúde, 2012; 19(3):182-187.

5. BRASIL. Lei 9.836, de 23 de setembro de 1999. Acrescenta dispositivos à Lei no 8.080 , de 19 de setembro de 1990, que "dispõe sobre as condições para a promoção, proteção e recuperação da saúde, a organização e o funcionamento dos serviços correspondentes e dá outras providências", instituindo o Subsistema de Atenção à Saúde Indígena. Diário Oficial da República Federativa do Brasil, Brasília, 23 de setembro de 1999. Capítulo V.

6. BRASIL. Ministério da Educação. Conselho Nacional de Educação. Câmara de Educação Superior. Resolução № 3, de 20 de Junho de 2014. Institui Diretrizes Curriculares Nacionais do Curso de Graduação em Medicina e dá outras providências. 2014 [citado em 15 de junho de 2017]. Disponível em: http://www.fmb.unesp.br/Home/Graduacao/resolucao-dcn-2014.pdf

7. BRASIL. Ministério da Educação. Conselho Nacional de Educação. Câmara de Educação Superior. Parecer CNE/CES no 116/2014, aprovado em 3 de abril de 2014. Diretrizes Curriculares Nacionais do Curso de Graduação em Medicina. 2014 [Citado em 06 de junho de 2017]. Disponível em: http://portal.mec.gov.br/index.php?option=com_docman\&view=download\&alias=15514-pces 116 -

14\&category_slug=abril-2014-pdf\&ltemid=3019̄2

8. BRASIL. Ministério da Educação. Conselho Nacional de educação. Resolução CNE/CES № 4, de 7 de novembro de 2001. Institui Diretrizes Curriculares Nacionais do Curso de Graduação em Medicina. 2001; [citado em 06 de junho de 2017]. Disponível em: http://portal.mec.gov.br/cne/arquivos/pdf/CES04.pdf

9. BRASIL. Ministério da Saúde. Portaria 290, de 28 de fevereiro de 2013. Institui o Componente Construção de Unidades Básicas de Saúde Fluviais no âmbito do Programa de Requalificação de Unidades Básicas de Saúde (UBS) aos Estados e aos Municípios da Amazônia Legal e Pantanal Sul Mato-grossense. Diário Oficial da União; 2013 [citado em 20 de maio 2017]. Disponível: http://sintse.tse.jus.br/documentos/2013/Mar/1/portaria-no-290-de-28-de-fevereiro-de-2013

10. BRASIL. Ministério da Saúde. Portaria № 2.866, de 2 de dezembro de 2011. Institui, no âmbito do Sistema Único de Saúde (SUS), a Política Nacional de Saúde Integral das Populações do Campo e da Floresta (PNSIPCF). Diário Oficial da União; 2011. [citado em 20 de maio 2017]. Disponível: http://bvsms.saude.gov.br/bvs/saudelegis/gm/2011/prt2866_02_12_2011.html

11. BRASIL. Ministério da Saúde. Portaria № 992, de 13 de maio de 2009. Institui a Política Nacional de Saúde Integral da População Negra. Diário Oficial da União; 2015 [citado $\begin{array}{lllll}\text { em } & 20 & \text { de } & \text { maio } & \text { 2017]. }\end{array}$ http://www.saude.go.gov.br/public/media/EU6sWLAaw55isy/61991121004160061911.pdf

12. COCKS M. Biocultural diversity: moving beyond the realm of 'indigenous' and 'local'people. Human Ecology, 2006; 34 (2):185-200.

13. COSTA DAS, SILVA RFD, LIMA VV, RIBEIRO ECO. Diretrizes curriculares nacionais das profissões da Saúde 2001-2004: análise à luz das teorias de desenvolvimento curricular. Interface-Comunicação, Saúde, Educação. 2018; 22(67): 1183-1195.

14. CRESWELL JW. Projeto de pesquisa: Métodos qualitativo, quantitativo e misto. 2nd ed. Porto Alegre: Artmed, 2007.

15. DE CASTRO NJC. O ensino da saúde indígena nos currículos e espaços acadêmicos. Ensino, Saude e Ambiente. 2015;8(1):15-25.

16. DE CASTRO NJC, CAVALCANTE IMS, PALHETA ADS, SANTOS DDN. Inclusão de disciplinas em graduação de enfermagem sobre populações tradicionais amazônicas. Cogitare Enfermagem, 2017; 22(2):e49730.

17. IBGE - Instituto Brasileiro de Geografia e Estatística. Censo 2010: população indígena é de 896,9 mil, tem 305 etnias e fala 274 idiomas. 2012; [citado em 02 junho 2015]. Disponível: http://saladeimprensa.ibge.gov.br/noticias. html?view=noticia\&id=1\&idnoticia=2194\&busca=1\&t=censo-2010populacao-indigena-896-9-mil-tem-305-etnias-fala-274.

18. LIMA VV, KOMATSU RS, PADILHA RQ. Desafios ao desenvolvimento de um currículo inovador: a experiência da Faculdade de Medicina de Marília. Interface-comunicação, saúde, educação, 2003; 7: 175-184.

19. SANTOS IWS. Organização curricular baseada em competência na educação médica. Revista Brasileira de Educação Médica, 2011; 35(1):86-92.

20. SILVA RP, BARCELOS AC, LUZ HBQ, SOTTOMAIOR IR, SILVA CJM, OLIVEIRA ST. A experiência de alunos do PET-Saúde com a saúde indígena e o programa Mais Médicos. Interface, 2015; 19(1):1005-1014.

21. SIMONIAN LTL. Uma relação que se amplia: fotografia, ciência sobre e na Amazônia. In: Kahwage, C; Ruggeri, S. Imagem e pesquisa na Amazônia: ferramentas de compreensão da realidade. Belém: Alves Gráfica e editora, 2007; p.17-24.

22. YIN RK. Estudo de caso: planejamento e métodos. 5aㅗ ed. São Paulo: Bookman; 2015. 